\title{
Energy Spectrum and Eigenfunctions through the Quantum Section Method
}

\author{
J.S. Espinoza Ortiz* and R. Egydio de Carvalho ${ }^{\dagger}$ \\ Instituto de Geociências e Ciências Exatas \\ Universidade Estadual Paulista
}

Rua 10, 2527, Santana, 13500-230, Rio Claro, SP, Brazil.

Received on 16 May, 2001. Revised version received on 23 July, 2001.

\begin{abstract}
We present the Quantum Section Method as a quantization technique to compute the eigenvalues and the eigenfunctions of quantum systems. As an instructive example we apply this procedure to quantize the annular billiard. The method uses the symmetry of the system to determine an auxiliary section separating the system into partial regions and computes the Green's functions for Schroedinger's equation, obeying the same boundary conditions imposed on the eigenfunctions of the system. The eigenvalues are obtained as zeroes of a finite real determinant and the eigenfunctions are also determined. The present analytical and numerical results are in total agreement with those obtained by other procedures, which shows the efficiency of the method.
\end{abstract}

\section{Introduction}

The Green's function method applied to the Schroedinger's equation associated to the Poincaré's section technique used to study classical dynamics, have originated a method to compute the eigenvalues and the eigenfunctions of quantum systems. This method is denoted by Quantum Section Method (QSM). It was first introduced by Bogolmony as an essential ingredient to the resummation theory of classical periodic orbits contributing to the quantum energy spectrum of chaotic systems $[1,2]$.

The connection between classical periodic orbits and quantum energy spectrum comes from the fact that the semiclassical limit of stationary states should be connected with classical invariant manifolds and for a chaotic Hamiltonian system the latter are the periodic orbits, besides the total energy. For systems which are not fully chaotic but mixed by chaos and regular motion, the quantum energy spectrum carries also influences of invariant tori.

A class of models which nicely describe regular, mixed or chaotic dynamics are the billiards. These correspond to geometrical boundaries where a particle moves freely inside them and reflects specularly at the boundaries. For these type of systems the motion is strongly dependent on the shape of the boundaries thus allowing the simulation of any desired dynamics.

In this work we consider the annular billiard (annulus) probing the applicability of the QSM. This billiard consists of two eccentric circles where only the annular region constitutes the accessible space to the motion. Changing the eccentricity we can control the sea of chaos and also the regularity of the system. We consider classical and quantum mechanical aspects of the annular billiard in section II. In section III we develop the QSM. Finally in section IV we present the numerical calculations and a discussion of these results.

\section{The Model}

As depicted in Fig. (1a-1b) the billiard is defined by two eccentric circles on the $X Y$-plane, where the outer circle has radius ' $R$ ' and the inner circle radius ' $r$ '. The centre of the outer circle is considered as the origin of the system and the inner circle can dislocate horizontally in relation to it. The distance between the centres of the circles is called by eccentricity and we denote it by ' $\delta$ ' . As ' $r$ ' as ' $\delta$ ' are constrained by the inequality $(r+\delta) \leq R$. The trajectories on the billiard are polygonal lines among collisions with the boundaries. There are two different types of motion identified by the letters 'A' and 'B' and when composed can describe any possible behavior of the system [3]. The A-type motion corresponds to those trajectories which collide with the outer circle before a collision with itself,

\footnotetext{
*e-mail: ortiz@rc.unesp.br

†e-mail: regydio@rc.unesp.br
} 
while the B-type motion reports to the trajectories colliding with the inner circle between two collisions with the outer one. There is also a particular case of Atrajectories, called Whispering Gallery Orbits (WGO), which never attain the inner circle, what means they never cross the caustic - an auxiliary circle with radius $(r+\delta)$. The caustic helps us to separate both kind of motions and later we will associate to it an algebraic tangency condition to distinguish the A-motion from the B-motion.
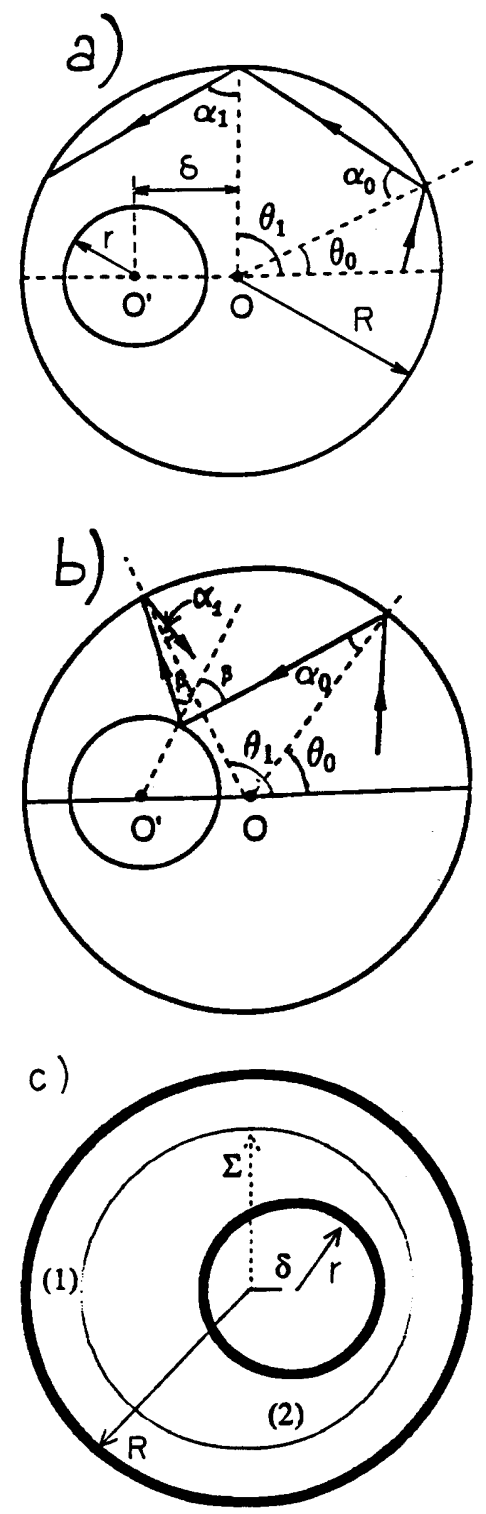

Figure 1. a) The annular billiard scheme exhibiting the outer circle of radius $R$, the inner circle of radius $r$, the eccentricity $\delta$, both angle coordinates $\theta$ and $\alpha$, and a typical trajectory of A-motion; b) a typical trajectory of B-motion; c) an auxiliary circular section of radius $\Sigma$, concentric with the inner circle, defining two partial regions.

The dynamics on phase space is naturally described by Birkhoff's coordinates, these are defined on the outer boundary at the positions where the collisions have occurred. Because of the circular geome- try, two angle coordinates suffice to define the collision points: ' $\theta$ ' associated with the arc-length counted from the positive abscissa axis and ' $\alpha$ ', the reflected angle formed between the trajectory and the normal at the collision point, see Fig. (1a-1b). Hence we define the phase space coordinates as $S=\sin (\alpha)$ and $L=\theta /(2 \pi)$, so that phase space reduces to the rectangle : $-1 \leq S \leq 1,-1 / 2 \leq L \leq 1 / 2$. Thus the motion can be described by a discrete dynamics, or merely a map, involving only kicks with the external circle. The map can be easily obtained through pure geometrical considerations. Hereafter we consider $R=1$ and for a trajectory starting at any initial condition $\left(\theta_{0}, \alpha_{0}\right)$ we require the tangency condition

$$
|\sin (\alpha)+\delta \sin (\alpha-\theta)|>r
$$

which means that at the next collision with the outer circle the trajectory had not previously hit the inner circle. This defines the $A$-motion (Fig. 1a) given by the map equations

$$
\begin{aligned}
& \alpha_{1}=\alpha_{0} \\
& \theta_{1}=\theta_{0}+\left(\pi-2 \alpha_{0}\right) .
\end{aligned}
$$

If the tangency condition is not satisfied, which means that at the next collision with the outer circle the trajectory has previously reached the inner circle once, the $B$-motion (Fig. 1b) is defined via the map equations

$$
\begin{aligned}
\sin (\beta) & =\left(\sin \left(\alpha_{0}\right)+\delta \sin \left(\alpha_{0}-\theta_{0}\right)\right) / r, \\
\left(\alpha_{1}+\theta_{1}\right) & =2 \beta-\left(\alpha_{0}-\theta_{0}\right) \\
\sin \left(\alpha_{1}\right) & =r \sin (\beta)-\delta \sin \left(\alpha_{1}+\theta_{1}\right),
\end{aligned}
$$

where $\beta$ is the angle of the reflection at the inner circle (see Fig. 1b). It is easy to verify from the first formula above as the tangency condition emerges. To generate the dynamics of a trajectory on phase space, the tangency condition should be checked at each iteration to select what map has to be used. The set of Figs. (2a-2d) exhibits the dynamic richness of the annular billiard depending on the relationship between $r$ and $\delta$. From these plots we can observe some general results [3], the origin of the phase space $(L, S)=(0,0)$ corresponds to an elliptic fixed point (stable equilibrium) while $r>\delta$ and it becomes a hyperbolic fixed point (unstable equilibrium) if $r<\delta$ passing through a neutral stability when $r=\delta$. Looking at the Fig. 1 b the trajectory $(L, S)=(0,0)$ corresponds to a line over the positive abscissa starting at the outer circle and kicking toward the inner circle. On the other hand we note that the point $(L, S)=( \pm 1 / 2,0)$ always corresponds to a hyperbolic fixed point (except when $\delta=0$ ). From Fig. 1b, the corresponding trajectory starts on the negative abscissa at the outer circle and kicks toward the inner circle. Numerical observations indicates that the accessible phase space becomes almost fully chaotic at $\delta \approx 3 r[3]$. In that context it is important to establish one more identification of the WGO: on phase space they are the straight lines which have ordinates $|S|>(r+\delta)$, consequently only in the region between the WGO's may occur chaos and resonance structures. 
Figure $(2 a)$

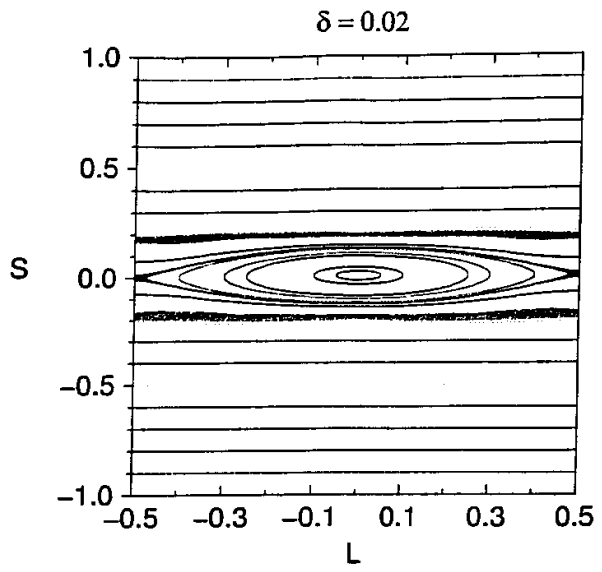

Figure $(2 b)$

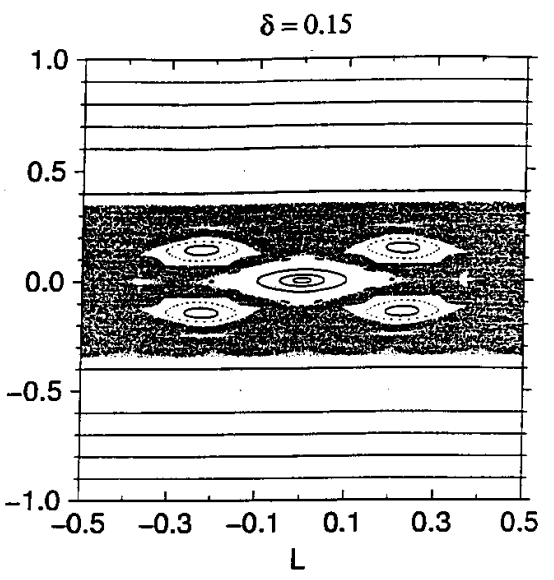

Figure (2c)

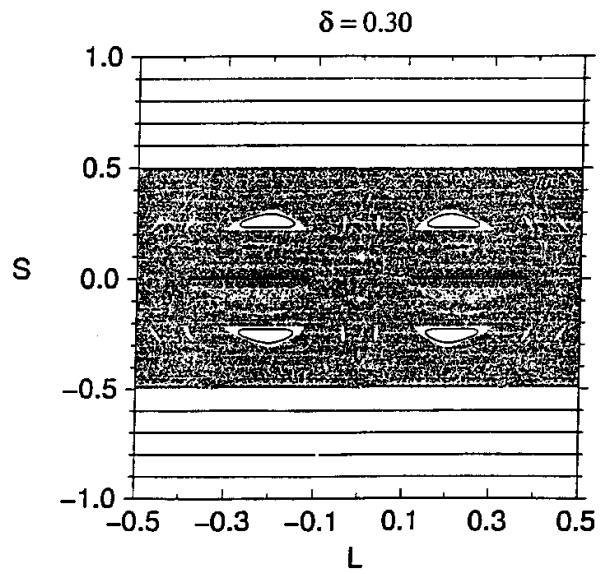

Figure (2d)

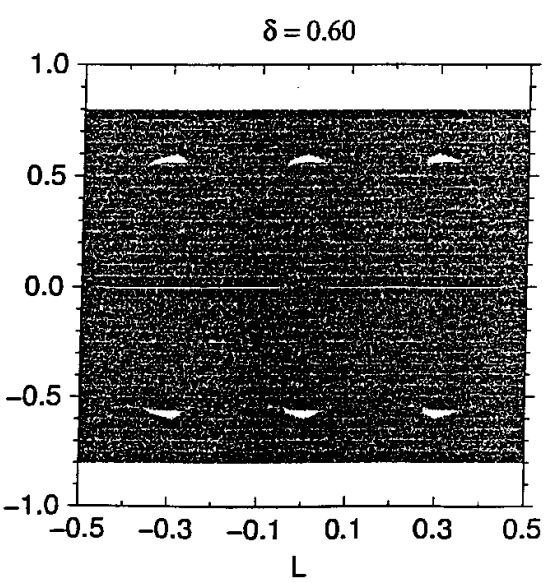

Figure 2. The phase space $(L, S)$ for $r=0.2$ and four values of $\delta$ : (a) $\delta=0.02$, there is a thin layer of chaos and the WGO are located at $|S|>0.22$; (b) $\delta=0.15$, the origin still corresponds to a stable fixed point but the chaotic sea has increased, the WGO are located at $|S|>0.35$; (c) $\delta=0.30$, the origin is a hyperbolic fixed point and chaos continues increasing, the WGO are located at $|S|>0.5$; (d) $\delta=3 r=0.60$, the accessible phase space is almost fully chaotic, and the WGO are located at $|S|>0.8$.

Regarding these main classical aspects of the annulus, the corresponding quantum problem consists on solving the Helmholtz equation

$$
\left\{\nabla^{2}+\kappa^{2}\right\} \Psi(\rho, \theta)=0,
$$

obeying Dirichlet's boundary conditions, where $\kappa=$ $\sqrt{2 E} / \hbar(m=1)$ with $\rho$ and $\theta$ the radial and angular polar variables respectively. It is analogous to the eigenmode problem for an annular vibrating membrane, also with an infinite number of discrete vibrational frequencies. For the case $\delta=0$ the eigenvalues $\kappa_{n m}$ associated with the Helmholtz's equation are obtained as zeroes of the equation

$$
\left(J_{n}\left(\kappa_{n m} R\right)-\frac{J_{n}\left(\kappa_{n m} r\right)}{Y_{n}\left(\kappa_{n m} r\right)} Y_{n}\left(\kappa_{n m} R\right)\right)=0
$$

and the corresponding eigenfunctions are given by

$$
\Psi_{n m}(\rho, \theta)=A_{n m}\left(J_{n}\left(\kappa_{n m} \rho\right)-\frac{J_{n}\left(\kappa_{n m} r\right)}{Y_{n}\left(\kappa_{n m} r\right)} Y_{n}\left(\kappa_{n m} \rho\right)\right) \times \begin{cases}\cos (n \theta) & , n=0,1,2, \ldots \ldots \text { even states } \\ \sin (n \theta) & , n=1,2,3, \ldots \ldots \text { odd states }\end{cases}
$$


where $J_{n}$ and $Y_{n}$ are the Bessel's functions of first and second kind respectively and $A_{n, m}$ is a normalization constant. Except for $n=0$ which is a single state, the remaining states are degenerate doublets. For the case $\delta \neq 0$ the angular momentum is not conserved, however the system still possesses a discrete symmetry under reflection with respect to the line joining the centres of both circles. Consequently the eigenstates of eq.(4) continue to be separated into even and odd states, but not longer degenerate due to an effect of 'dynamical tunnelling' which splits them. This phenomena, influenced by chaos, is attributed to classical transport connecting WGO regions [3, 4]. These quantum billiard has been considered by different theoretical methods in the literature [5, 6, 7, 8] and also experimentally [9], paving the directions for us to apply the QSM.

\section{The Quantum Section Method}

This method considers the possibility to separate any system into partial regions through one auxiliary section in order to explore certain local symmetries, as for example billiard boundaries or potential symmetries (in the case of open systems) [10].

The uncertainty principle precludes the simultaneous knowledge of momenta and position of a quantum particle, hence the quantum section is developed only on position space. We construct partial Green's functions, $G_{1}\left(q, q^{\prime} ; E\right)$ and $G_{2}\left(q, q^{\prime} ; E\right)$, in both partial regions with the following properties: a) they satisfy the non-homogeneous Schroedinger's equation inside each partial region

$$
\left\{\nabla^{2}+\kappa^{2}\right\} G_{j}\left(q_{j} q_{j}^{\prime} ; \kappa\right)=\delta\left(q_{j}-q_{j}^{\prime}\right) ; j=1,2 ;
$$

b)on the boundaries these Green's functions should satisfy the same boundary conditions as the eigenfunctions of the original system, c) these Green's functions may be arbitrary on the section, but they should match continuously. We write down the ansatz for solutions in the form of a single layer potential

$$
\Psi_{j, E}(q)=\int G_{j}\left(q, Q^{\prime} ; E\right) \mu_{j}\left(Q^{\prime}\right) d Q^{\prime}
$$

where $Q^{\prime}$ is defined on the section and $\mu_{j}$ is the single layer density which is initially an unknown function. The idea now is to take a solution in one partial region and extend it to the other region, imposing continuity for the partial functions $\Psi_{j, E}(q)$ and their derivatives on the section. Then the quantization condition emerges and the eigenvalues are obtained as zeroes of a matrix determinant defined on the section. This allows the single layer density to be obtained and further to calculate the eigenfunctions of the original system.

For the annular billiard the additional partial separability of the Schroedinger's equation in polar coordinates makes possible to construct exact partial Green's functions in eigenmodes representation and from there the construction of precise partial functions in each side of the section as depicted in Fig.1c. We define the section as an auxiliary circle of radius $\Sigma$, concentric with the outer circle, which separates both partial regions: the region (1) outer to the ' $\Sigma$ circle 'and the region (2) inner to it.

The Green's functions are constructed satisfying the non-homogeneous Helmholtz's equation (7), where $q_{1}=(\rho, \theta)$ and $q_{2}=(\zeta, \phi)$ are the positions in relation to the ' $R$-circle' and the' $r$-circle', respectively. As for the eigenfunctions, we require $G_{1}$ to satisfy Dirichlet's boundary conditions on the outer circle. In the same fashion $G_{2}$ cancels on the inner circle, with both Green's functions arbitrary over the section.

Due to partial separability in polar coordinates in relation to the 'R $(r)$ circle', we may write

$$
\begin{array}{lll}
G_{1}\left(\rho \rho^{\prime}, \theta \theta^{\prime} ; \kappa\right) & =\sum_{m} g_{1, m}\left(\rho \rho^{\prime} ; \kappa\right)\langle\theta \mid m\rangle\left\langle m \mid \theta^{\prime}\right\rangle ; & \rho^{\prime}<\rho<R, \\
G_{2}\left(\zeta^{\prime} \zeta, \phi^{\prime} \phi ; \kappa\right)=\sum_{n} g_{2, n}\left(\zeta^{\prime} \zeta, \kappa\right)\langle\phi \mid n\rangle\left\langle n \mid \phi^{\prime}\right\rangle ; & r<\zeta<\zeta^{\prime}
\end{array}
$$

where $\langle m \mid \theta\rangle=\langle\theta \mid m\rangle \propto \cos [\sin ](m \theta)$ for the Green's function $G_{1}$ and for the Green's function $G_{2}$ one exchanges $m \rightarrow n$ and $\theta \rightarrow \phi$ to obtain $\langle n \mid \phi\rangle=\langle\phi \mid n\rangle \propto \cos [\sin ](n \phi)$. For integers $m$ or $n$ the angular functions ' $\cos ^{\prime}$ and $^{\prime} \sin ^{\prime}$ will yield solutions possessing even and odd symmetries, respectively. Now it is chosen a particular one-dimensional Green's function $g_{j}$ in radial coordinates, excluding any possibility for $G_{1}$ and $G_{2}$ to cancel over the section

$$
\begin{aligned}
& g_{1, m}\left(\rho \rho^{\prime} ; \kappa\right)=H_{m}^{(1)}(\kappa R) H_{m}^{(2)}\left(\kappa \rho^{\prime}\right)\left\{Y_{m}(\kappa R) J_{m}(\kappa \rho)-J_{m}(\kappa R) Y_{m}(\kappa \rho)\right\} \\
& g_{2, n}\left(\zeta^{\prime} \zeta ; \kappa\right)=H_{n}^{(1)}\left(\kappa \zeta^{\prime}\right) H_{n}^{(2)}(\kappa r)\left\{Y_{n}(\kappa r) J_{n}(\kappa \zeta)-J_{n}(\kappa r) Y_{n}(\kappa \zeta)\right\}
\end{aligned}
$$


where $J, Y, H^{(1,2)}$ are the Bessel functions of the first, second and third kind respectively. These Green's functions are given, in Appendix A, by applying the image method for a source in $\rho^{\prime}\left(\zeta^{\prime}\right)$ with respect to $\rho=R$ $(\zeta=r)$ in such a way that the quantum dynamics corresponds to two quantum paths between $\rho^{\prime}$ and $\rho\left(\zeta^{\prime}\right.$ and $\zeta)$. One path is direct while the other reflects in $R(r)$. There is no possibility for the particle to come back since it leaves the section. In this sense there is an analogy with the scattering formalism[11].

Both Green's functions are diagonal in each eigenmodes representation. We write down any given partial wave function in each partial region in the form of a single layer density

$$
\begin{aligned}
& \Psi_{1}(\rho, \theta ; \kappa)=\int_{0}^{2 \pi} d \theta \prime \mu\left(\theta^{\prime}\right) G_{1}\left(\rho \rho^{\prime}, \theta \theta^{\prime} ; \kappa\right), \\
& \Psi_{2}(\zeta, \phi ; \kappa)=\int_{0}^{2 \pi} d \phi^{\prime} \mu\left(\phi^{\prime}\right) G_{2}\left(\zeta^{\prime} \zeta, \phi^{\prime} \phi ; \kappa\right) .
\end{aligned}
$$

These functions satisfy Schroedinger's equation with appropriated boundary conditions in each partial region. The condition for these functions and their normal derivatives to match over the section is the existence of only one density. This condition imposes

$$
\int_{0}^{2 \pi} d \theta^{\prime} \mu\left(\theta^{\prime}\right) \tilde{G}\left(q_{2}^{\prime} q_{1}^{\prime} ; \kappa\right)=\int_{0}^{2 \pi} \tilde{G}\left(q_{2}^{\prime} q_{1}^{\prime} ; \kappa\right) \mu\left(\phi^{\prime}\right) d \phi^{\prime}=0
$$

where

$$
\tilde{G}\left(q_{2}^{\prime} q_{1}^{\prime} ; \kappa\right)=\int_{0}^{2 \pi} d \theta\left\{G_{2}\left(\zeta^{\prime} \zeta, \phi^{\prime} \phi ; \kappa\right) \partial_{\rho} G_{1}\left(\rho \rho^{\prime}, \theta \theta^{\prime} ; \kappa\right)-G_{1}\left(\rho \rho^{\prime}, \theta \theta^{\prime} ; \kappa\right) \partial_{\rho} G_{2}\left(\zeta^{\prime} \zeta, \phi^{\prime} \phi ; \kappa\right)\right\} .
$$

This equation may be rewritten as

$$
\tilde{G}\left(q_{2}^{\prime} q_{1}^{\prime} ; \kappa\right)=\sum_{m n}<\phi^{\prime}|n><n| \tilde{G}|m><m| \theta^{\prime}>
$$

where ,

$$
\begin{aligned}
<n|\tilde{G}| m> & =\int_{0}^{2 \pi} d \theta\left\{G_{1}(\rho, \theta ; \kappa) \partial_{\rho} G_{2}(\zeta(\rho, \delta, \theta), \phi(\rho, \delta, \theta) ; \kappa)+\right. \\
& \left.-G_{2}(\zeta(\rho, \delta, \theta), \phi(\rho, \delta, \theta) ; \kappa) \partial_{\rho} G_{1}(\rho, \theta ; \kappa)\right\},
\end{aligned}
$$

and the condition given in eq.(12) becomes

$$
\sum_{m}\langle\mu \mid m\rangle\langle m|\tilde{G}| n\rangle=\sum_{n}\langle m|\tilde{G}| n\rangle\langle n \mid \mu\rangle=0 .
$$

The eigenvalues are computed as the zeroes of $\operatorname{det}|\langle n|\tilde{G}| m\rangle|$, where the integrals are evaluated over the section. Before computing the integrals it is necessary to drive $\rho^{\prime}$ onto $R$ as well as $\zeta^{\prime}$ onto $r$ and to expand $G_{2}(\zeta, \phi)$ in terms of $(\rho, \theta)$. This expansion uses the addition theorem for Bessel's functions

$$
J_{n}(\kappa \zeta)\left[\begin{array}{c}
\cos (n \phi) \\
\sin (n \phi)
\end{array}\right]=\cos (n \theta) \sum_{l} J_{l+n}(\kappa \rho) J_{l}(\kappa \delta)\left[\begin{array}{c}
\cos (l \theta) \\
\sin (l \theta)
\end{array}\right] \pm \sin (n \theta) \sum_{l} J_{l+n}(\kappa \rho) J_{l}(\kappa \delta)\left[\begin{array}{c}
\sin (l \theta) \\
\cos (l \theta)
\end{array}\right],
$$

and

$$
Y_{n}(\kappa \zeta)\left[\begin{array}{c}
\cos (n \phi) \\
\sin (n \phi)
\end{array}\right]=\cos (n \theta) \sum_{l} Y_{l+n}(\kappa \rho) J_{l}(\kappa \delta)\left[\begin{array}{c}
\cos (l \theta) \\
\sin (l \theta)
\end{array}\right] \mp \sin (n \theta) \sum_{l} Y_{l+n}(\kappa \rho) J_{l}(\kappa \delta)\left[\begin{array}{c}
\sin (l \theta) \\
\cos (l \theta)
\end{array}\right]
$$

Finally the integrals are computed on the section considering

$$
\begin{aligned}
\int_{0}^{2 \pi} \cos (n \theta) \cos (m \theta) \cos (l \theta) & =\left(\delta_{l,-(m-n)}+\delta_{l,(m+n)}+\delta_{l,(m-n)}+\delta_{l,-(m+n)}\right) / 4 \\
\int_{0}^{2 \pi} \sin (n \theta) \cos (m \theta) \sin (l \theta) & =\left(\delta_{l,(m+n)}+\delta_{l,-(m-n)}-\delta_{l,(m-n)}-\delta_{l,-(m+n)}\right) / 4 \\
\int_{0}^{2 \pi} \cos (n \theta) \sin (m \theta) \sin (l \theta) & =\left(\delta_{l,(m+n)}+\delta_{l,(m-n)}-\delta_{l,-(m-n)}-\delta_{l,-(m+n)}\right) / 4 \\
\int_{0}^{2 \pi} \sin (n \theta) \sin (m \theta) \cos (l \theta) & =\left(\delta_{l,-(m-n)}+\delta_{l,(m-n)}-\delta_{l,(m+n)}-\delta_{l,-(m+n)}\right) / 4
\end{aligned}
$$

yielding the goal

$$
\begin{aligned}
<n|\tilde{G}| m> & =\frac{1}{2}\left\{\left[Y_{n}(\kappa r) J_{m}(\kappa \rho)-J_{n}(\kappa r) Y_{m}(\kappa \rho)\right] \partial_{\rho}\left[Y_{m}(\kappa R) J_{m}(\kappa \rho)-J_{m}(\kappa R) Y_{m}(\kappa \rho)\right]\right. \\
& \left.-\left[Y_{m}(\kappa R) J_{m}(\kappa \rho)-J_{m}(\kappa R) Y_{m}(\kappa \rho)\right] \partial_{\rho}\left[Y_{n}(\kappa r) J_{m}(\kappa \rho)-J_{n}(\kappa r) Y_{m}(\kappa \rho)\right]\right\}\left.\right|_{\rho=\Sigma} \\
& \times\left\{J_{m-n}(\kappa \delta) \pm(-1)^{n} J_{m+n}(\kappa \delta)\right\} .
\end{aligned}
$$


In this form the eigenvalues are obtained as zeroes of the determinant of the real matrix given in eq.(20), where the \pm refers to the even and odd solutions, respectively.

\section{Results and Discussion}

Without loss of generality by taking the section over to the outer circle, what reads $\Sigma=R$, the matching condition leads to the known Nagaya \& Sing \& Kothary's eigenvalues expression[5]. The case $\delta=0$ implies that the single possibility providing non-trivial solutions for eq.(20) holds when $m=n$. In this case the matrices become diagonal for both parities and the eigenvalues are exactly degenerate (except when $m$ and $n$ are null). When $\kappa \delta>|m \pm n|$, for a fixed $\kappa$, the Bessel's functions become non-oscillating and they decrease exponentially as $|m \pm n|$ increases. So it is necessary to be careful when enlarging the determinant dimension because it will become numerically unstable. Hence as a nice and interesting consequence, the dimension of $\operatorname{det}\{\langle n|\tilde{G}| m\rangle\}$ is always finite. With the eigenvalues in hand the eigenfunctions are readily determined going back to eq.(16) and computing the density $\mu$.

The operator given in eq.(14) is called quantum transfer operator and in the semiclassical limit it can be expressed in term of classical paths providing a way to interpret the quantum dynamical tunnelling phenomena in terms of classical transport.

We have computed the eigenvalues with 14 digits of precision for any given energy scale. Figs. (3) and (4) concern to an energy spectrum with the following set of parameters: $\hbar \approx 0.4583, r=0.40$ and $\delta=0.19$. In Fig.(3) it is plotted the numerical cumulative density of states for the first 2500 eigenvalues and in Fig.(4) its deviation in relation to the soft cumulative density of states for billiards, given by the formula: $N(\kappa)=\frac{1}{4 \pi \hbar^{2}}\left\{A \kappa^{2}-L \kappa\right\}$ [12], where for the annular billiard, $A$ is the net area $(=\pi(R+r)(R-r))$ and $L$ the net perimeter $(=2 \pi(R+r))$. From both plots it is inferred that no level is missing from the energy spectrum and that there is no lack of convergence. A sequence of eigenvalues of both parities together is shown in Fig.(5) for different values of the eccentricity, with fixed $\hbar \approx 0.2731$, and $r=0.20$. This plot presents the levels dynamics as a function of $\delta$ exhibiting 'avoided crossing' as it is expected in chaotic/mixed systems. Note also the degeneracies breakdown for some levels as chaos increases (remember the chaotic sea enlarges with the increasing of $\delta$, see Fig.(2a-2d)). These energy splittings relating levels of uncoupled parities are attributed to a kind of tunneling denoted as dynamical tunneling. Finally some scarred eigenfunctions are presented in Fig.(6) for some selected eigenvalues obtained with the same parameters of Fig.(3). They carry information of the classical dynamics and they correspond to trajectories lying in different positions on phase space.

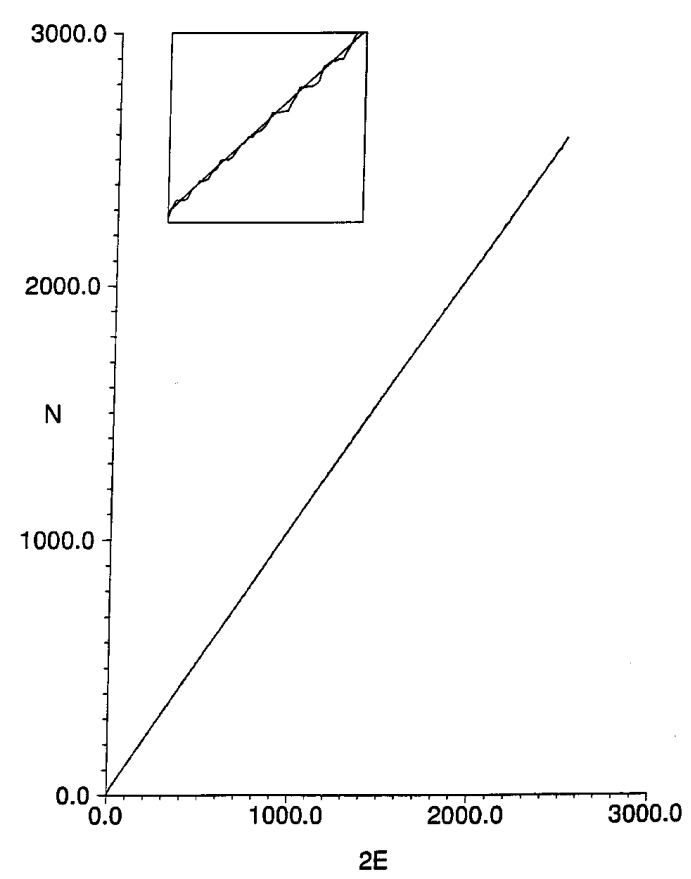

Figure 3. The numerical cumulative density of states for the first 2500 eigenvalues using the set of parameters: $\hbar \approx$ $0.4583, r=0.40$ and $\delta=0.19$. The insert picture shows the numerical cumulative density of states and the soft cumulative density of states for billiards.

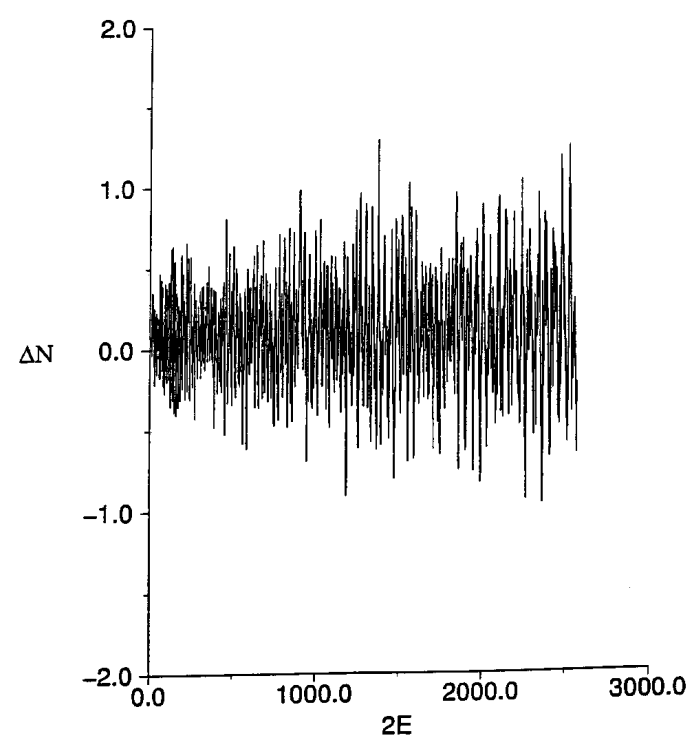

Figure 4. The deviation $(\Delta N=(N)$ numerical $-(N)$ soft $)$ between the numerical cumulative density of states and the soft cumulative density of states using the same set of parameters of Fig.(3). Both cumulative densities differ around one unity in module what means a very good agreement between them. 


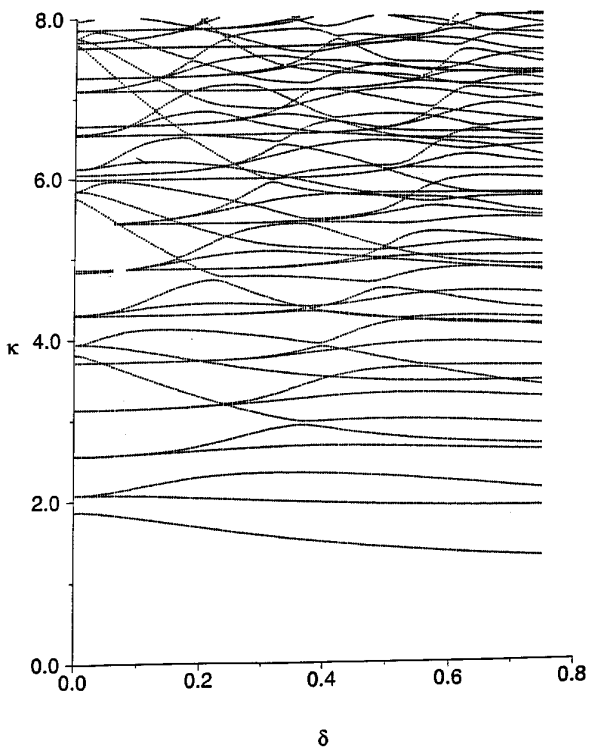

Figure 5. The levels quantum dynamics. A sequence of eigenvalues is shown for different values of the eccentricity using the following set of parameters $\hbar \approx 0.2731, r=0.20$ and $\delta=[0.0,0.75]$.
Finally these quantum analytical and numerical results fit completely with the known results concerning this billiard as established in the literature and essentially they assert that the QSM is an efficient quantization method.

\section{Acknowledgments}

We thank the Brazilian scientific agencies Fundação de Amparo a Pesquisa do Estado de São Paulo (FAPESP) and Fundação para o Desenvolvimento da Unesp (Fundunesp) for financial support. JSEO is particularly acknowledged to the LPTMS at Orsay Cedex, France for kind hospitality during last visit and to Dr. D. Ullmo for fruitful discussions.

\section{Appendix - The Radial Green Functions}

Here it is considered the partial region 2 where the partial radial Green's function is constructed with the following boundary conditions: it is null at $\zeta=r$ and it is arbitrary at $\zeta=\zeta^{\prime}$. These conditions lead to the definitions

$$
\begin{array}{llll}
g_{2}\left(\zeta \zeta^{\prime} ; \kappa\right) & =\mathcal{A}\left\{Y_{n}(\kappa r) J_{n}(\kappa \zeta)-J_{n}(\kappa r) Y_{n}(\kappa \zeta)\right\} & & ; r<\zeta<{ }^{\prime} \\
\tilde{g}_{2}\left(\zeta \zeta^{\prime} ; \kappa\right) & =\mathcal{B}\left\{J_{n}(\kappa \zeta)+i Y_{n}(\kappa \zeta)\right\} & ; \zeta^{\prime}<\zeta,
\end{array}
$$

where $\mathcal{A}$ and $\mathcal{B}$ are constants to be determined from the additional restrictions at $\zeta=\zeta^{\prime}$

$$
\begin{aligned}
g_{2}\left(\zeta \zeta^{\prime} ; \kappa\right)-\left.\tilde{g}_{2}\left(\zeta \zeta^{\prime} ; \kappa\right)\right|_{\zeta=\zeta^{\prime}} & =0, \\
\left.\frac{1}{\zeta} \frac{d}{d \zeta}\left\{g_{2}\left(\zeta \zeta^{\prime} ; \kappa\right)-\tilde{g}_{2}\left(\zeta \zeta^{\prime} ; \kappa\right)\right\}\right|_{\zeta=\zeta^{\prime}} & =-1 .
\end{aligned}
$$

Solving eq.(A-2) yelds,

$$
g_{2}\left(\zeta \zeta^{\prime} ; \kappa\right)=H_{n}^{(1)}\left(\kappa \zeta^{\prime}\right) H_{n}^{(2)}(\kappa r)\left\{Y_{n}(\kappa r) J_{n}(\kappa \zeta)-J_{n}(\kappa r) Y_{n}(\kappa \zeta)\right\}, \quad r<\zeta<\zeta^{\prime} .
$$

By similar arguments the Green's function in the partial region (1) is given by

$$
g_{1}\left(\rho \rho^{\prime} ; \kappa\right)=H_{n}^{(1)}(\kappa R) H_{n}^{(2)}\left(\kappa \rho^{\prime}\right)\left\{Y_{n}(\kappa R) J_{n}(\kappa \rho)-J_{n}(\kappa R) Y_{n}(\kappa \rho)\right\}, \quad \rho^{\prime}<R<R .
$$

\section{References}

[1] E. Bogomolny, Comments Atomic Molecular Phys. 2567 (1990); and in Nonlinearity 5, 1055 (1992).

[2] E.B. Bogomolny and J.P. Keating, Phys. Rev. Lett. 77, $1472(1996)$

[3] O. Bohigas, D. Bosé, R. Egydio de Carvalho and V. Marvulle, Nucl. Phys. A 560. 197 (1993).

[4] O. Bohigas, S. Tomsovic and D. Ullmo, Phys. Rev. Lett. 64, 1479 (1990); and in Phys. Rep. 233, 43 (1993).

[5] K. Nagaya, J. Sound Vib. 50, 545 (1977).

[6] G.S. Singh and L.S. Kothari, J. Math. Phys. 25, 810 (1984).
[7] M.J. Hine, J. Sound Vib. 15, 295 (1971).

[8] S.D. Frischat and E. Doron, Phys. Rev. E 57, 1421 (1998).

[9] C. Dembowisk, H.D. Graf, A. Heine, R. Hofferbert, H. Rehfeld and A. Richter, Phys. Rev. Lett. (2000).

[10] J.S. Espinoza Ortiz and A.M. Ozorio de Almeida, J. Phys. A 30, 7301 (1997); and in Physica D 145 (2000).

[11] E. Doron and U. Smilansky, Nonlinearity 5, 1055 (1992).

[12] H.P. Baltes and E.R. Hilf, Spectra of Finite Systems, (Bibliographisches Institutes, Mannheim, 1975) 
a)

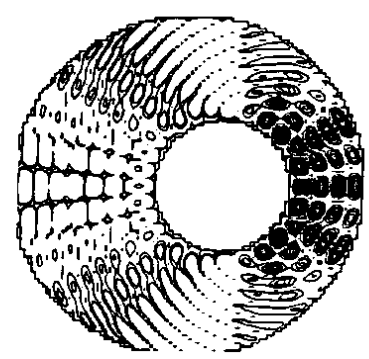

c)

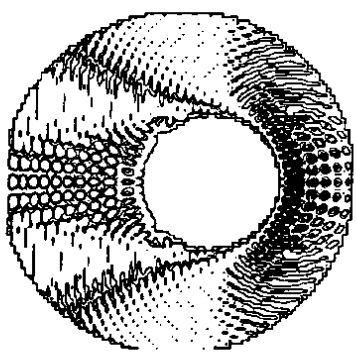

e)

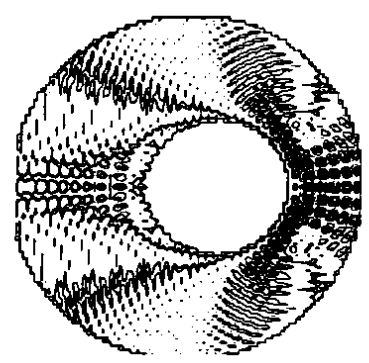

g)

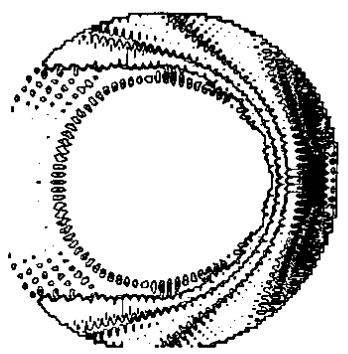

i)

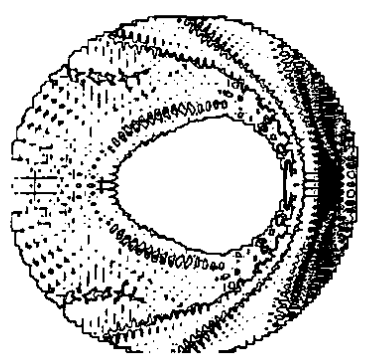

b)

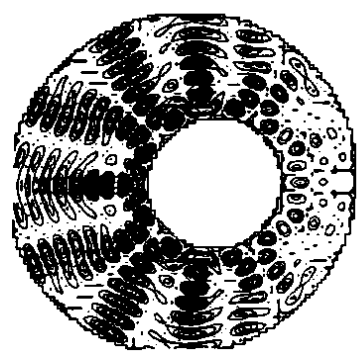

d)

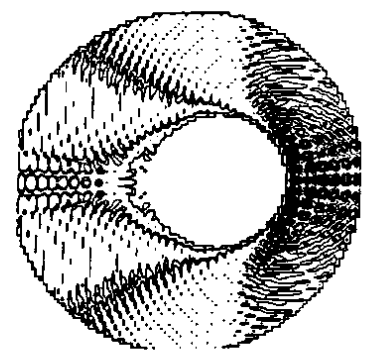

f)

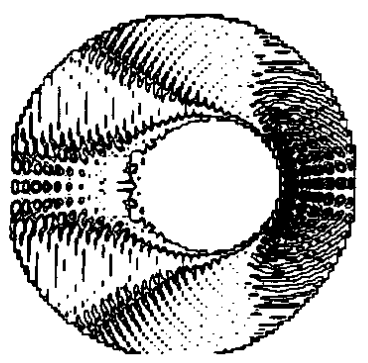

h)

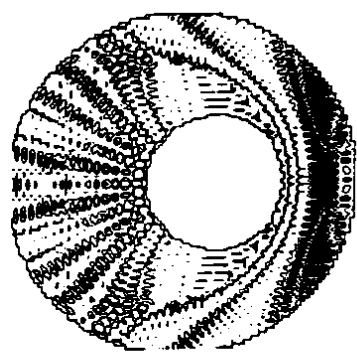

j)

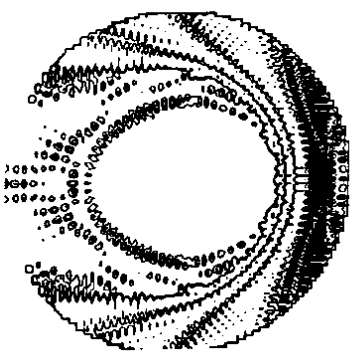

Figure 6. Scarred eigenfunctions using the same set of parameters of Fig.(3). The even solutions are located on the left of the figure and the odd solutions are located on the right. The eigenvalues used in the plots are the following: a) $\kappa=16.8961439227900, b) \kappa=16.91942974911557, c) \kappa=27.3224162938150, d) \kappa=27.3035930847495, e) \kappa=$ $27.4114121918047, f) \kappa=27.3452473498958, g) \kappa=31.6387754925558, h) \kappa=31.6383421881693, i) \kappa=31.7006255714796$, j) $\kappa=31.6448142075546$ 